\title{
Youth's Presentation of Themselves on Instagram and Facebook with respect to Body image focusing on appearance
}

\author{
Jill Mehta ${ }^{1}$, Aasiya Ghoghari ${ }^{2}$, Vaibhavi Naik ${ }^{3}$ \\ ${ }^{1,2,3}$ D. G. Ruparel College of Arts, Science and Commerce \\ Corresponding author: Jill Mehta \\ Email-jillmehta39@gmail.com
}

\begin{abstract}
This study was done to investigate youth's presentation of themselves on Instagram and Facebook. Participants (N-266) were told to fill an online questionnaire which consisted of 31 multiple choice questions with choice of answers differing from question to question. Youths were studied on frequency of posting pictures, appearance related things done before or while posting pictures, their expectations from others, comparison and engagement on Instagram and Facebook. It was observed that participants use filters to enhance their looks in the pictures. Participants reported that they do not feel pressured to look good in their posts and they don't compare themselves with others. The amount of engagement in these platforms increases after posting pictures of themselves to check all reaction a post gets.
\end{abstract}

Keywords: Instagram, Facebook, youth, body image, appearance.

(Paper received $-3^{\text {rd }}$ July 2019, Peer review completed $-6^{\text {th }}$ September 2019)

(Accepted $-12^{\text {th }}$ September 2019)

\section{INTRODUCTION}

There are lot of social and cultural factors that affects person's body image. Society often constructs our behaviours and beliefs, such as personal developments, physiological and psychological interactions, and the common perception of our bodies as a reflection of self-worth. Body image struggles have been prevalent for many centuries now, with the rapid constant shifts in ideal body types. In the past, norms were typically set by cultural beliefs, genders, or social factors. Despite these being prevalent today, changes in the fashion and media industries are other influences at hand [1]. On account of the evolution of society today, text messaging, emails, social media, and other technological advancements have dominated people's face to face interactions. Although technology provides the convenience of connecting with others easily, its association with the media has resulted in a "platform of delivery in which we intercept and interpret messages about ourselves, our self-worth, and our bodies"[2].

The phrase body image was first coined by the Austrian neurologist and psychoanalyst Paul Schilder (1935) in his book 'The Image and Appearance of the Human Body (1935)' [3].

The Oxford dictionary meaning of body image is "The subjective concept of one's physical appearance based on self-observation and reactions of others". Another definition says, "Body image is how one views themselves in the mirror or in their mind. It incorporates memories, experiences, assumptions, comparison of one's own appearance and overall attitudes towards their height, shape and weight" [4].

An individual's impression of their body and appearance is also assumed to be a product of ideals on social media. Majority of the users choose to create a positively biased self-presentation [5].

Today in the world of internet, social media platforms like Instagram and Facebook are most popular among Indians with 201 million Facebook users and 46 million Instagram users of which $90 \%$ of Facebook and $85 \%$ of Instagram users are under the age of 45 years [6]. 
Instagram and Facebook are platforms that allow users to share personal photos, videos and information due its popularity in personal photo sharing among young people. These photos and videos may be used as appearance feedback seeking platforms. In case of photos, this often consists of posting 'idealized' images through the use of camera angle, filters, digital manipulation software like Photoshop; developing a personality identity through self presentation has been cited as an important aspect of social media [7]. Social media lets anyone enter the beauty pageant. Teens can cover up pimples, whiten teeth and even airbrush with the swipe of a finger, curating their own image to become prettier, thinner and hotter.

All this provides an illusion of control: "If I spend more time and really work at it, I can improve at being beautiful". "I don't get to choose how I'm going to leave my apartment today," one young woman told. "If I could, my body would look different. But I can choose which picture makes my arms look thinner" [8]. Several studies have been done in relation with social media and body image. A study found that cultural pressures via media may lead to development of negative body image (body image and eating-disorder). Media images of 'thin' females and 'muscular' males represent idealized version of physical attractiveness [9]. Study on Irish females revealed that women are aware that they are experiencing negative body image self-impression because they try to imitate the models they see in digital media [10]. Similar studies have been done on effects on body image by social media's, T.V.'s effect on eating disorders. However, there are limited studies on how social media affects appearance related comparisons, expectations and assumptions. The data relevant to these studies is even more limited in the Indian context.

\section{METHODOLOGY}

This study attempts to investigate youth's frequency of posting pictures, things done before or while posting pictures, expectations, comparison and engagement on Instagram and Facebook. Method

\section{Participants}

Participants $(\mathrm{N}=266)$ were from the age group of 15 to 24 years $(\mathrm{M}=18.82)$ of which 142 were females $(\mathrm{M}=18.37)$ and 124 were males $(\mathrm{M}=19.25)$. Participants were from different regions of Mumbai. Participants who said that they never post their own pictures on Facebook and Instagram were excluded (18 participants).

\section{Material}

A self prepared questionnaire including 31 questions mainly concentrating on following four aspects:

i. Frequency of being engaged in photo sharing on Instagram and Facebook.

ii. Appearance related things done before or while posting pictures on Instagram and Facebook.

iii. Consequences if any (like comparison, pressure to look good, etc.) while posting picture on Instagram and Facebook.

iv. Frequency of checking reactions after posting their own pictures on Instagram and Facebook. The questions were multiple choice questions with choice of answers differing from question to question.

\section{Procedure}

The survey was done online using Google Forms; it was based on random participation. The Google form link was sent to various people through WhatsApp and email. Consent of participants was taken at the beginning of the form. Each form of the survey took about 15-20 minutes for the participants to fill. After filling of all the forms by the participants, question-wise analysis was done of the responses recorded.

\section{RESULTS}

All the participants (N-266) used Instagram or Facebook, and majority of them used both (72.9\%). All the participants were those who posted pictures on these platforms. It was observed that $36.8 \%$ of the participants change their profile picture once in three months and $35 \%$ of the participants post their pictures monthly. The common reason given for posting pictures was to let others know more about themselves $(39.5 \%)$. Some other reasons were to feel more acceptable in the virtual world $(19.9 \%)$, to assure that even 
they are updating themselves with the modernizing world (32\%), to get more people to like and comment on them through the pictures (8.6\%). $75 \%$ participants use filters to enhance their looks in the pictures. $53.4 \%$ participants said they do not use cosmetic/Grooming products on themselves to look good in pictures. More than half of the participants said they give importance to their looks in the picture more than occasion or people in the pictures $(57.2 \%)$.

Participants reported that they did not compare themselves with others (74. 5\%) or feel pressured to look good in the pictures (54\%) nor do they plan their looks in advance (50\%). They did not spend time thinking about looks while posting pictures. (41.7\%). Participants also said that they give same amount of importance to physical appearance even if they are definitely not going to post the picture.

Majority of the participants check their Instagram and Facebook more frequently after posting pictures (66.9\%). Participants also expected certain people to like or comment upon their picture (53.4\%) and also check who all have liked their post (55.2\%). 33.9\% boys accepted that they go to gymnasium to do exercise or follow diet to look good in the pictures whereas only $5.0 \%$ of the females accepted it.

\section{DISCUSSION}

The survey was done concentrating on four major aspects which are:

i. To find out how often people engage in photo sharing themselves on Instagram and Facebook.

ii. Appearance related things people do before or while posting the pictures.

iii. Consequences if any (like comparison, pressure to look good, etc.) while posting picture.

iv. iv) Frequency of checking reactions after posting their own pictures on Instagram and Facebook.

It was found that posting pictures is common in participants than changing profile picture, major reason for posting was letting others know more about themselves. In doing so, it is seen that participants do not use cosmetic/grooming products on themselves before taking the picture (50.3\%) but the need to look good is still there which is satisfied by apps. $75 \%$ of the participants accepted using filters to enhance their looks in the picture. In a UK based study on males found $57 \%$ boys feel pressured by social media to look good(Ads add to boys' body-image pressure, 2016) and in a survey done by dove, it was found that pressures from social media doubles for adolescent girls between the ages of 13 to 18 years old [11]. Socio-cultural model puts forward that society promotes physical attractiveness and beauty for both males and females through social media. This is in alignment with the results of the study as the need to attain these beauty standards is clearly seen.

These networks are effective medium and more celebrated in 21st century and are believed to manifest episodes of real life. In this manifestation, people use filters to portray oneself in appealing manner. Participants do not use cosmetic/grooming before taking the picture, which reflects that these apps itself are designed in such a way that it replaces and satisfies the need of such products. The enlarged sense of media personalization and tendency to use the Internet for social purposes has led millennials to get increasingly invested in developing an idealized online self that they can present to the world [5-7].

Participants also reported that they give same amount of importance to physical appearance even if they are definitely not going to post the picture. Though participants said that they did not feel pressured to look good or think about looks while posting, $75 \%$ of them do use filters to enhance their looks in the pictures. Hence, there is discrepancy in what they say and how they behave. Possibly same is the case of real life and virtual world. Studies have also shown that perhaps comparison does take place on social media through self enhancing posts. People upload self-enhancing post and see such contents from others on their feeds. This could lead to social comparison with oneself and with these images and feel inferior [12].

More than half of the participants (66.6\%) agreed that they check who have liked their post. 53.4\%. participants expect specific people (such as friends, family etc.) to like and comment on their post. 55.3\% participants check who all have liked their post. Recent study discovered a strong connection between Facebook and the brain's reward centre, called the nucleus accumbens. This area processes rewarding 
feelings about things like food, sex, money and social acceptance. The feeling on receiving positive feedback lights up this accumbens part of the brain. This is likely to get an individual hooked to these platforms [13]. Certain amount of difference was found between boys and girls when asked if they went to a gymnasium or followed diet to look good in the pictures. $33.9 \%$ boys accepted that they go to gymnasium or follow diet to look good in the pictures whereas only $5.0 \%$ of the females accepted it. The importance men placed on muscle and weight may be traced back to the 'bulked-up action heroes', along with the brawny characters in many video games, present an anatomically impossible ideal for boys, much as Barbie promotes proportions that are physically impossible for girls The main causes of male body issues include $25 \%$ being teased about their weight, while $33 \%$ specified social media as the source for self-consciousness [14]. The pursuit of an idealised online self could however provide a dangerous playground for adolescents and young adults who may end up with low self esteem and negative body image. Often people feel isolated and rejected when their expectations are not fulfilled.

\section{CONCLUSION}

There were no major discrepancies found in the replies of males and females. So, it can be said that social media affects both the genders in nearly equal manner. However, if we take a positive look, the figures which indicate influence of social media on youth are still average and not on very higher sides. Also, some other figures like $74 \%$ participants do not compare themselves with others, 54\% participants do not feel pressured to look good in the pictures, etc show that still there is light, social media has not completely overpowered youth. This study acknowledges requirement of further research in this area with regards to differences in response of different age groups, cultures, economic status and also if factors like self-esteem is affected along with any other negative consequences.

\section{Practical implication and suggestions}

In today's age of filters, people are bombarded with idealized beauty images and people seeking social approval through social media, there is need to help youth go beyond their appearance and to assess their own strengths. As looks are temporary, virtues are permanent. At larger level, there is a huge need to reach out to children and youths through institutions like schools and colleges; training students, parents and teachers to emphasise on health, hygiene, neatness and skill set rather than merely external looks; and surmise to identify themselves more with their blue ribbons (high qualities) rather than transitory looks.

\section{REFERENCES}

1. Markova I, Azocar C. The influence of social media and fashion magazines on body image of culturally and racially diverse young women. Retrieved from www.lib.dr.iastate.edu

2. Reddy S, Otieno R. Relationship between body image and clothing perceptions: Among women aged 18-55 years in the UK. Int J Arts Comm 2013;2(5):40-9.

3. Schilder P. The image and appearance of the human body. Routledge; 2013.

4. Holland G, Tiggemann M. A systematic review of the impact of the use of social networking sites on body image and disordered eating outcomes. Body Image 2016;17:100-10.

5. Lupinetti VM. Self-presentation and social media: A qualitative examination of the use of Instagram by amateur NPC female figure competitors. Thesis retrieved from https://scholarworks.sjsu.edu/etd

6. Kwatra N. Why Instagram has become the next Facebook? Mint. 2018.

7. Manago AM, Graham MB, Greenfield PM, Salimkhan G. Self-presentation and gender on MySpace. J Appl Dev Psychol 2008;29(6):446-58.

8. Preston C, Ehrsson HH. Illusory changes in body size modulate body satisfaction in a way that is related to non-clinical eating disorder psychopathology. PloS One 2014;9(1):e85773.

9. Jones DC. Social comparison and body image: Attractiveness comparisons to models and peers among adolescent girls and boys. Sex Roles 2001;45(9-10):645-64.

10. Behan E. Negative Reflections-How Social Media Affects Body Image Satisfaction in Irish Females. Doctoral dissertation, Dublin, National College of Ireland; 2018. 
11. Ridgway JL, Clayton RB. Instagram unfiltered: Exploring associations of body image satisfaction, Instagram\# selfie posting, and negative romantic relationship outcomes. Cyberpsychol Behav Soc Networking 2016;19(1):2-7.

12. Uhlir JL. Social comparison and self-presentation on social media as predictors of depressive symptoms. Retrieved from http://scholarship.claremont.edu/scripps_theses/756; 2018.

13. Somerville LH. The teenage brain: Sensitivity to social evaluation. Curr Dir Psychol Sci 2013;22(2):121-7.

14. Aubrey JS. The impact of sexually objectifying media exposure on negative body emotions and sexual selfperceptions: Investigating the mediating role of body self-consciousness. Mass Commun Soc 2007;10(1):1-23.

\author{
Acknowledgements - Nil \\ Conflict of Interest - Nil; \\ Funding - Nil
}

